\title{
Full Endoscopic Transforaminal Lumbar Discectomy: A Literature Review
}

\author{
Guan-Chyuan Wang ${ }^{1}$, Chien-Min Chen ${ }^{2,3,4}$ \\ ${ }^{1}$ Department of Neurosurgery, Hualien Tzu Chi Hospital, Buddhist Tzu Chi Medical Foundation, Hualien, Taiwan, R.O.C \\ ${ }^{2}$ Division of Neurosurgery, Department of Surgery, Changhua Christian Hospital, Changhua, Taiwan \\ ${ }^{3}$ School of Medicine, Kaohsiung Medical University, Kaohsiung, Taiwan, R.O.C \\ ${ }^{4}$ College of Nursing and Health Sciences, Dayeh University, Taiwan, R.O.C
}

$-$

Corresponding Author:

Chien-Min Chen, MD, PhD

Division of Neurosurgery, Department of Surgery, Changhua Christian Hospital, 135 Nanxiao St., Changhua-city, Changhua-county 500, Taiwan

Tel: $+88-64-723-8595$

Fax: +88-64-722-8289

E-mail: 96015@cch.org.tw

Received: March 03, 2021

Revised: March 15, 2021

Accepted: March 18, 2021
Since its introduction in 1973, Kambin's triangle had made endoscopic transforaminal lumbar discectomy (TELD) possible. Dr. Yeung further developed a complete spinal endoscope system and approach methods (the Yeung endoscopic spine system). With the advancement of endoscopic instruments such as the high-speed burr, reamer, and trephine, Dr. Hoogland introduced foraminoplasty methods to extract herniated disks. Since then, the surgical field of TELD has been broadly widened. Many interesting and innovative surgical methods have been developed to overcome the limitations of TELD, such as managing the migrating disk or operation at the L5/S1 level. In this article, we describe the surgical steps of TELD and discuss relevant literatures associated with it.

Key Words: Endoscope, Minimally invasive surgery, Spine

\section{INTRODUCTION}

Disk herniation is the protrusion, extrusion, or sequestration of the nucleus pulposus. When the herniated disk compresses the neural structure and cause symptoms, discectomy is indicated. The surgical technique has evolved from open surgery to minimally invasive methods. The concept and goal of minimally invasive discectomy are to minimize the length of the surgical incision and destruction of muscles and bony structures.

\section{History}

After its introduction in 1973 by Dr. Parviz Kambin, Kambin's triangle has made endoscopic discectomy at the lumbar area possible ${ }^{1,12,15)}$. The transforaminal route through the Kambin's triangle (the safe working zone) was then widely accepted". Two main endoscopic transforaminal discectomy techniques were proposed by Drs. Yeung and Hoogland ${ }^{14,311}$ In 2005, Dr. Ruetten introduced the endoscopic interlaminar approach that facilitates discectomy at the L5/S1 level owing to the high iliac crest and caudal sequestrating disk beyond the pedicle ${ }^{25,26}$. With the development of advanced spinal endoscopic instruments, especially the multiple- diameter Kerrison punches, special reamers, trephines, bone-biting forceps, high-speed/angular burrs, and larger work- ing sheaths (working channel of $5.5 \mathrm{~mm}$ as compared with 2.7-4.2 $\mathrm{mm})$, the scope of endoscopic spinal surgery has largely expanded $^{\text {t }}$. Though infrequently, endoscopic discectomy has been adop-

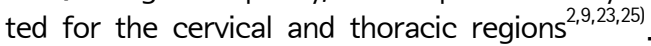

In this section, we review the surgical techniques of transforaminal endoscopic lumbar discectomy (TELD). We detail the patient position, skin marking, anesthesia, and operative procedure.

\section{Surgical Procedure}

\section{1) Patient Position and Skin Marking}

Patients were usually placed in the prone position. However, if the patient could not tolerate the prone position during local anesthesia (e.g., difficulty breathing or severe back pain during prone position), the lateral position is considered an option. To increase the intervertebral disk and foraminal space, flexing the patient's lumbar spine and hip is considered. Then, we draw skin markings to determine the entry point and insertion depth of the guiding needle. Traditionally, four lines are drawn on the patient's back, namely at the midline, anteroposterior inclination, lateral inclination, and insertion line (Figure 1). The anteroposterior view was used to locate the spinal level and to aim for the upper border of the pedicle, and the needle tip should not surpass one half of the pedicle (Figure 1A). The posterior end of the spinous processes determined the lateral aspect of the entry 
point (Figure 1B). Finally, we used a lateral view tos estimate the puncture depth (Figure 1B). Three lines are used to decide the surface entry point, and the midline is used for reference (Figure $1 \mathrm{C}$ ). The iliac crest should be drawn when performing the TELD at the L5/S1 level.

\section{2) Anesthesia and Skin Incision}

The transforaminal approach can usually be performed under local anesthesia (excluding intravenous drug sedation or pain control). The author performed an intermittent, step-by-step injection technique (Figure 2). First, the location of the nociceptor is located at the skin and the facet join. Therefore, these two places are the primary sources of pain during TELD. We used two 10-ml syringes for local injection. One was used to inject $1 \%$ pure lidocaine, and the another was used to inject $0.25 \%$ lidocaine. We used $1 \%$ lidocaine to infiltrate along the endoscopic tract, including the skin, muscle, and nearby facet joint. After reaching the target, we infiltrated it with $0.25 \%$ lidocaine at the foraminal space (Figure 2). The lidocaine dosage near the target was reduced to avoid completely blocking the motor fiber, including the nerve root. In our experience, $0.25 \%$ lidocaine would partially block the nerve root and preserve the sensory

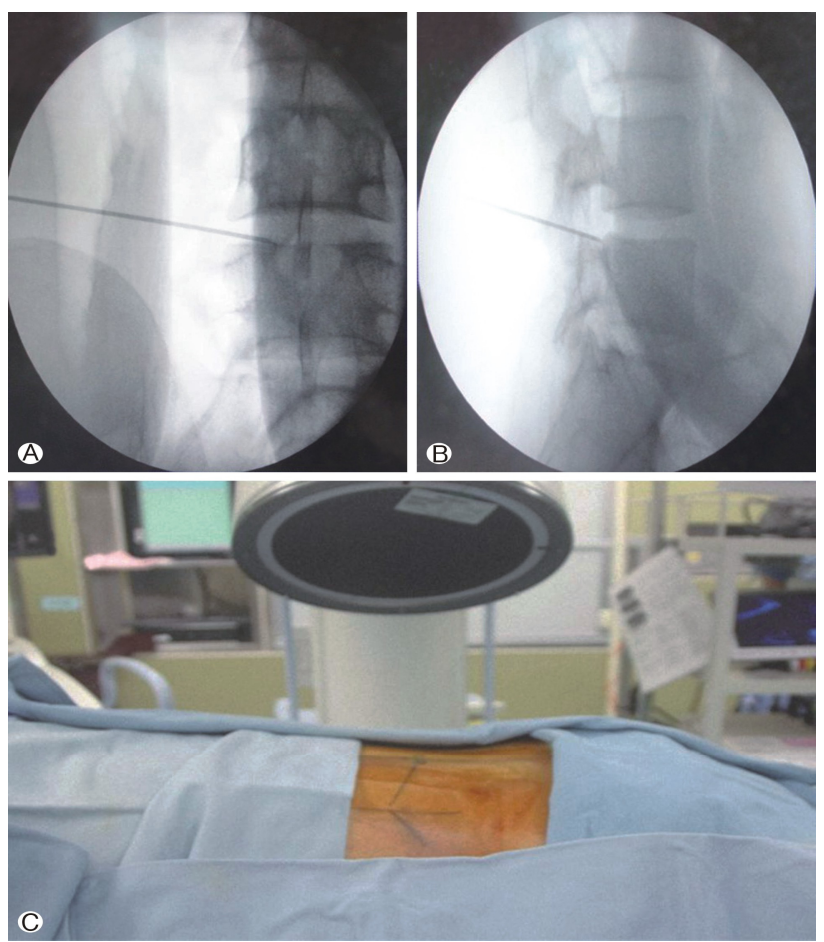

Figure 1. Localize the entry point. Under fluoroscopy, we located the surface entry point of the endoscopic transforaminal discectomy. (A): Anteroposterior view used to locate the spinal level and to aim for the upper border of the pedicle (green dashed line). (B): The posterior end of the spinous processes indicating the lateral aspect of the entry point (blue dashed line). We also used a lateral view to estimate the puncture depth (red dashed line). (C): The three lines are used to decide the surface entry point, and the midline is used as a reference (black dashed line). nerve. Patients could feel pain if the nerve roots are manipulated, which should serve as a warning signal to the surgeon.

\section{3) Insertion of the Endoscope}

We usually start the insertion of the guidewire at the anteroposterior fluoroscopic view. The guidewire was inserted in accordance with the skin marks. At around halfway to the target, the fluoroscope was changed to lateral view to check the depth of the guidewire. Then, the guidewire was advanced until touching the facet joint where we could feel a clear resistance. Then, we withdrew the guidewire a little bit and then increased the vertical inclination to enter the foraminal space. After insertion of the guidewire, the dilator was then introduced followed by the endoscopic sheath. Under fluoroscopy, the final docking point of the endoscopic sheath was checked. It should be located near the base of the pedicle in the lateral view (Figure $3 \mathrm{~A}$ ) and the midline of the pedicle in the anteroposterior view (Figure 3B).

\section{4) Surgical Procedure}

After proper placement of the endoscopic sheath, the endos-

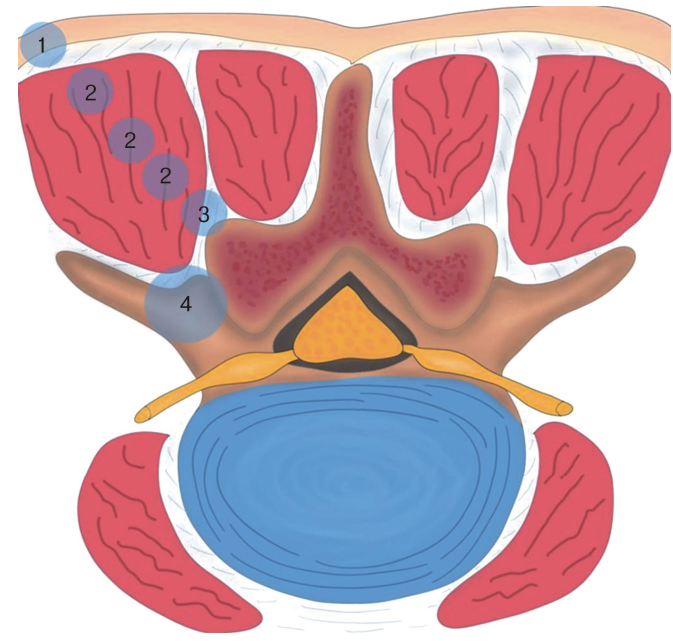

Figure 2. Applying the local anesthesia. Local anesthesia for transforaminal lumbar discectomy by applying 1\% lidocaine at the 1 . skin, 2. muscle, and 3. facet joint, and $0.25 \%$ lidocaine at the foraminal space.

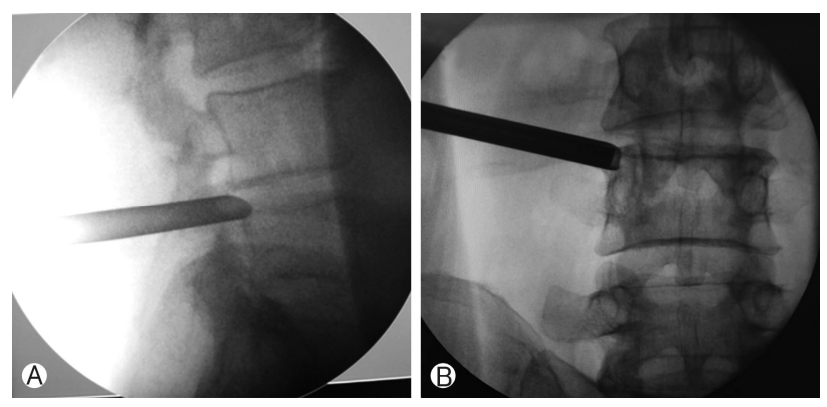

Figure 3. Final fluoroscopic view of the endoscopic sheath insertion (A): anteroposterior and (B): lateral views. 
cope (SPINENDOS GmbH, Munich, Germany) was then introduced. One camera and three ports were installed at the tip of the endoscope (Figure 4). The largest port was the working channel, while the two smaller ports were for irrigation and drainage. We used $0.9 \%$ saline for continuing irrigation. Some surgeons used a water pump, but we prefer using gravity to irrigate and free drainage without a pump. We suggested adjusting the pressure at the operative field to $30-50 \mathrm{mmHg}$ to control bleeding and prevent the increase of intracranial pressure. A high-speed burr (Vantage Technology Co., Ltd., Taoyuan, Taiwan), reamer, or trephine was used to perform foraminoplasty at the tip of the superior articular process or the base of the pedicle, which increased the working space and degree of freedom of the instruments (Figure 5). Endoscopic bipolar electrocoagulation (Vantage Technology Co., Ltd., Taoyuan, Taiwan) was used to control bleeding and coagulate small vessels before cutting them. Some ligaments and small vessels at the neural foramen were cut using punches and cutting scissors. If the herniated disks were at the foramina or far-lateral space, then they might be found at this stage and removed. The exiting nerve root should be carefully identified and protected. If the exiting nerve root is irritated, the patient would feel pain and respond to the surgeon (which was the main reason for performing TELD under local anesthesia rather than under general anesthesia). If the herniated disks were at the spinal canal, extended foraminotomy was indicated to enter the space. The spinal sac was identified, and the herniated disk could be removed with an endoscopic punch.

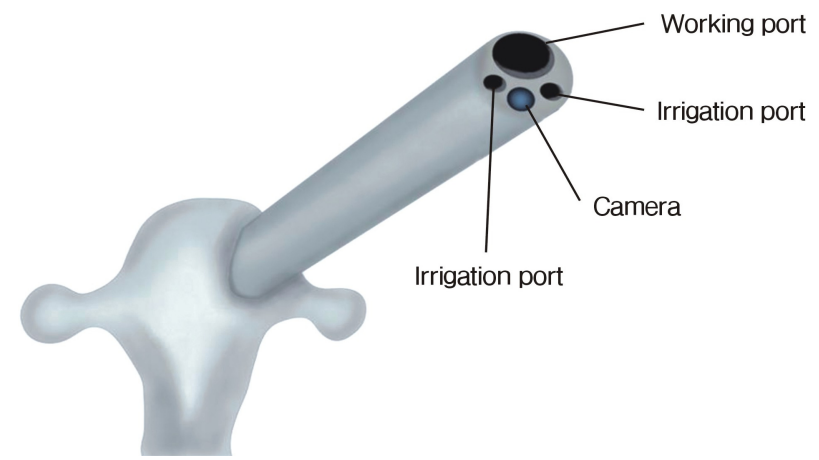

Figure 4. The channel of the endoscope consisting of four ports. The working port is for surgical instruments such as burrs, disk forceps, and punches. The two irrigation ports are for the passage of normal saline. The final port is for the camera.

\section{5) Final Checking Point}

The number of disks that should be removed is controversial. Previous studies on patients who underwent microdiscectomy showed that aggressive disk removal could reduce the recurrence rate but lower patient satisfication ${ }^{27}$. Therefore, surgeons should weigh between recurrence and patient satisfaction and decide when to end disk removal.

The final step of the surgery is to carefully check for bleeding. Some bleeders might be detected by using water pressure; when the irrigation is stopped, blood starts oozing. Therefore, after closing the irrigation system, bleeding must be controlled by radiofrequency ablation or absorbable hemostat. Oozing at the bone wedge could be controlled by bone wax or by using a highspeed diamond burr, which generates heat that helps coagulate bleeders from the cancellous bone. A hemostatic matrix such as Floseal (Baxter Medical, Fremont, California) or Surgiflo (Johnson \& Johnson Wound Management, a division of Ethicon Inc, Somerville, $\mathrm{NJ}$ ) was also beneficial to control the oozing at the surgical field.

\section{DISCUSSION}

\section{Two Concepts of TELD}

The transforaminal approach is based on two main concepts, one developed by Dr. Yeung (the Yeung endoscopic spine system [YESS] or inside-out technique) and the other developed by Dr. Hoogland (the intracanal transforaminal endoscopic surgical system [TESSYS] or outside-in technique) ${ }^{14,30,311}$. Although both are posterolateral approaches through the Kambin's triangle, they have some differences. The YESS technique punctures directly on the center of the disk and then pulls the herniated disk indirectly from the nucleus pulposus (Figure 6). On the other hand, the TESSYS tech- nique focuses on performing the foraminoplasty first to enlarge the working space and then advancing the instru-

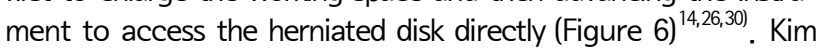
et al. further improved this technique into the mobile outside-in transforaminal approach and foraminoplasty, which overcame the limitations of the small neural foramen ${ }^{19}$. By applying this technique, the endoscopic view was usually called "half and half" because half of the view is the disk and the other half is the epidural space (Figure 7) ${ }^{19,21)}$. The advantages of the YESS techni-
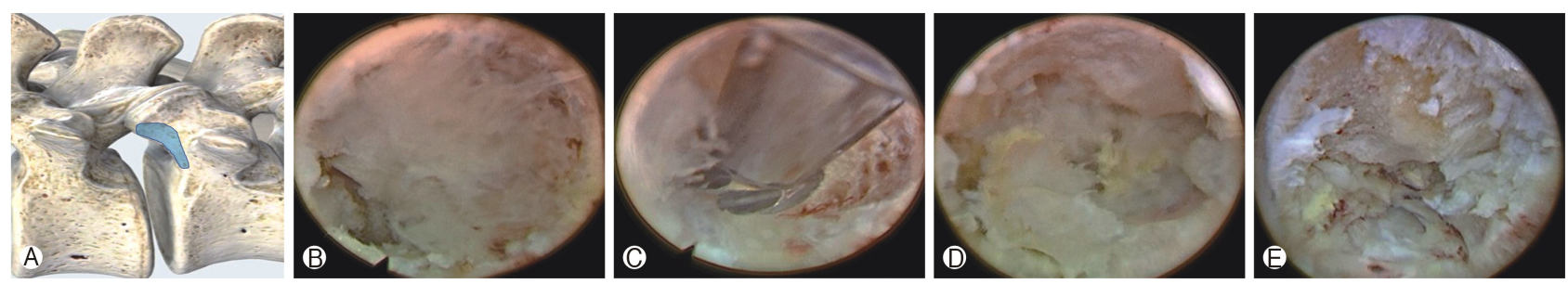

Figure 5. Foraminoplasty technique. Foraminoplasty is performed to increase the surgical freedom of the endoscopic sheath and instruments and to access the dural sac. (A): The blue area marks the extension of foraminoplasty, which includes the tip of the superior articular process and base of the pedicle. (B)-(E): Gradually enlarging the foramen and finally identifying the dural sac (asterisk). 


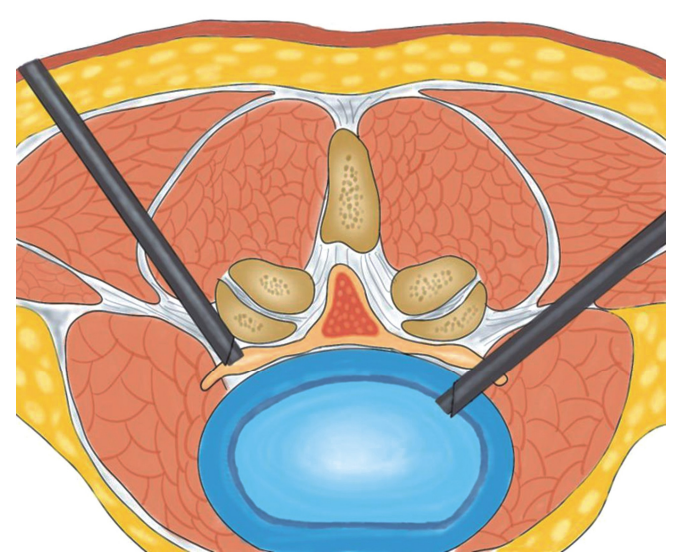

Figure 6. TESYS and YESS. On the left is the transforaminal endoscopic surgical system (TESSYS), or outside-in technique, and on the right is the Yeung endoscopic spine system (YESS), or insideout technique. For the TESSYS, the endoscope is docked at the foramen, and then foraminoplasty is performed. After the foramen is enlarged, the endoscope is advanced to find the herniated disk. For the YESS, the endoscope is directly inserted in the disk space and then the herniated disk is indirectly pulled out.
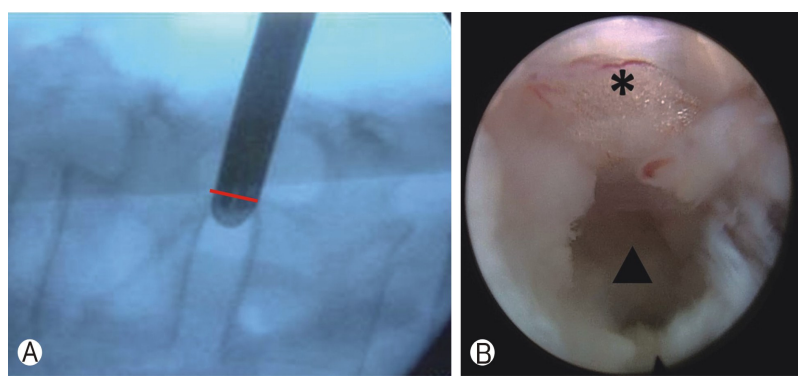

Figure 7. Half-and-half technique. Half-and-half view. (A): Half of the endoscopic sheath is in the disk, and half is in the foramen. (B): Under endoscopic view, both the spinal cord (asterisk) and disk (triangle) could be visualized. que were the reduced surgical time and more direct access to the disk space. However, the YESS technique might result in some residual disk (especially for the migrating disk) and a higher risk of damaging the neural structure ${ }^{30)}$. As the puncture site is more lateral and ventral, it could penetrate the abdominal cavity or retroperitoneal organs. On the other hand, the TESSYS technique is more popular nowadays because it is safer and causes less nerve injury, though potentially time-consuming and technically demanding ${ }^{30)}$.

\section{Limitations of TELD at the L5/S1 Level}

Transforaminal discectomy at the L5/S1 level was a challenge because the working channel was interfered by the iliac crest and had a narrow foraminal space ${ }^{7)}$. Some methods can be used to overcome this obstacle ${ }^{7,8)}$. Choi et al. classified the iliac crest height into six types and concluded that the transforaminal approach could be performed without difficulty when the iliac crest height is below the mid-L5 pedicle. On the other hand, if the iliac crest height is above the mid-L5 pedicle, foraminoplasty should be considered. Chen et al. depicted the details of the suprailiac approach for a high iliac crest. By inserting the working sheath in the intersection of the tangent line to the iliac crest and toward the superior articular process (SAP), it could be advanced and finally docked at the junction of the SAP and S1 pedicle. Then, the ventral part of the SAP was removed with an endoscopic burr or trephines. Foraminoplasty provided the view and space required to perform discectomy (Figure 8$)^{6}{ }^{6}$. The other surgical method is the transiliac approach. Choi et al. performed transiliac L5/S1 discectomy by trephining a hole at the iliac crest to pass the working sheath ${ }^{8)}$. The suprailiac approach has the advantage of obtaining adequate surgical freedom of the instruments, management of upward or downward migrating disk, and avoiding damaging vessels and nerves near the iliac bone. However, it could cause extensive facet joint destruction and is more time-consuming. On the contrary, the transiliac approach takes less time and pre-
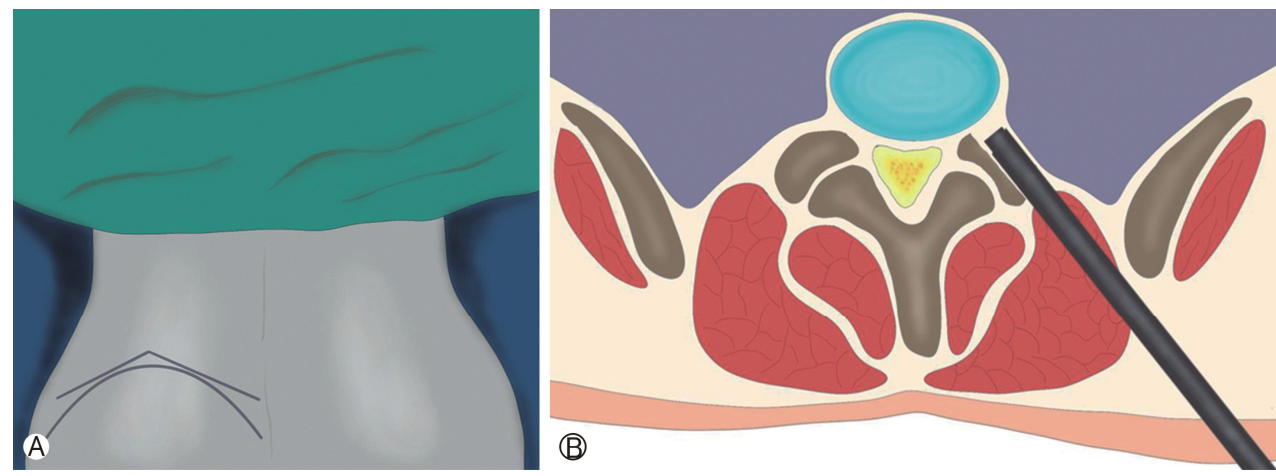

Figure 8. Management of the high iliac crest at L5/S1 level. Endoscopic lumbar discectomy for high iliac crest. (A): The iliac crest line was first marked, and two tangent line was then marked. The entry point was at the intersection of these two lines. (B): The endoscopic corridor was more caudal and medial compared to normal iliac crest or other lumbar levels; therefore, the initial surgical field was blocked by the superior articular process. The superior articular process and base of the pedicle must be partly removed to gain access to the spinal canal and herniated disc. 
serves the facet joint. However, the viewing angle and degree of freedom might be limited.

\section{Migrating Disk}

Migrating disk has been considered a relative contraindication of transforaminal discectomy, and an interlaminar approach is preferred ${ }^{9,22)}$. However, with the advancement of surgical instruments and techniques, it was no longer a limitation ${ }^{7}$. Chen et al. developed a surgical technique to deal with the downward migration of herniated disk in the transforaminal approach ${ }^{7}$. Kim et al. used a semi-rigid flexible probe to pull the migrating disk $^{18)}$. The technique included drilling the base of the SAP, part of the superior pedicle, and the vertebral body to widen the surgical field and gain access to the downward migrating disk. With endoscopic transforaminal discectomy, upward migration is easier to deal with than downward migration ${ }^{13,19}$. The key steps were to visually recognize the exiting root and to watch for the segmental artery to prevent nerve injury and retroperitoneal hematoma $^{19)}$.

\section{Recurrence}

Recurrent disk herniation is the main concern in not only endoscopic discectomy but also traditional laminotomy and microdiscectomy. Some of the identified risk factors included age, upper lumbar level, herniated disk type, disk degenerative grading (based on Pfirrmann's grading system), age, early ambulation, and body mass index $(\mathrm{BMI})^{7,20,28,32)}$. Kim et al. published an article on predicting early recurrence. They found that higher BMI, higher Pfirrmann disk degenerative grade, combined disk herniation, and early ambulation were risk factors of early recurrence ${ }^{20)}$. Chen et al. included 521 cases and found that older age, alcohol use, and higher lumbar level were risk factors of recurrence. Yin et al. conducted a meta-analysis and found that older age, obesity, upper lumbar level, and central disk herniation were risk factors of recurrence. Moreover, many studies have shown that the prolapsed type of herniated disk was associated with poorer surgical outcome but found no evidence of its association with recurrence.

Kim et al. proposed a technique to seal the annular tear by using radiofrequency to coagulate the circumference of the annulus defect ${ }^{16}$. Their results were promising in terms of decreasing the recurrence rate.

\section{CONCLUSION}

With the development of new techniques and instruments, the surgical spectrum of TELD has been largely widened. It is a fascinating technique because it involves not only a smaller wound and less tissue destruction but also a procedure that can be performed under local anesthesia and even at the outpatient clinic. In addition, it offers another surgical corridor to deal with herniated disks other than the posterior approach. Some limitations and obstacles remain to be overcome, and we are looking forward to seeing improvements in the future.

\section{CONFLICT OF INTEREST}

Dr. CM Chen is an editorial board member of the journal but was not involved in the peer reviewer selection, evaluation, or decision process of this article. There are no other potential conflicts of interest relevant to this article to declare.

\section{REFERENCES}

1. Ahn Y: Endoscopic spine discectomy: Indications and outcomes. International Orthopaedics 43(4):909-916, 2019 doi:10.1007/ s00264-018-04283-w

2. Ahn Y, Lee SH, Chung SE, Park HS, Shin SW: Percutaneous endoscopic cervical discectomy for discogenic cervical headache due to soft disc herniation. Neuroradiology 47(12):924-930, 2005 doi:10.1007/s00234-005-1436-y

3. Bae J, Chachan S, Shin SH, Lee SH: Transforaminal endoscopic thoracic discectomy with foraminoplasty for the treatment of thoracic disc herniation. Journal of Spine Surgery 6(2):397-404, 2020 doi:10.21037/jss.2019.11.19

4. Chen CM, Lin GX, Sharma S, Kim HS, Sun LW, Wu HH, Chen YC: Suprapedicular retrocorporeal technique of transforaminal full-endoscopic lumbar discectomy for highly downwardmigrated disc herniation. World Neurosurgery 143:e631-e639, 2020 doi:10.1016/j.wneu.2020.08.038

5. Chen CM, Sun LW, Tseng C, Chen YC, Wang GC: Surgical outcomes of full endoscopic spinal surgery for lumbar disc herniation over a 10-year period: A retrospective study. PLOS ONE, 15 (11):e0241494, 2020 doi:10.1371/journal.pone.0241494

6. Chen KT, Jabri H, Lokanath YK, Song MS, Kim JS: The evolution of interlaminar endoscopic spine surgery. Journal of Spine Surgery 6(2):502-512, 2020 doi:10.21037/jss.2019.10.06

7. Chen KT, Wei ST, Tseng C, Ousw S, LW, Chen CM: Transforaminal endoscopic lumbar discectomy for 15 -s1 disc herniation with high iliac crest: Technical note and preliminary series. Neurospine 17:S81-S87, 2020

8. Choi G, Kim JS, Lokhande P, Lee SH: Percutaneous endoscopic lumbar discectomy by transiliac approach: A case report. Spine (Phila Pa 1976), 34(12):E443-E446, 2009 doi:10.1097/BRS. 0b013e31817c4f39

9. Choi G, Prada N, Modi HN, Vasavada NB, Kim JS, Lee SH: Percutaneous endoscopic lumbar herniectomy for high-grade downmigrated L4-L5 disc through an L5-S1 interlaminar approach: A technical note. Minimally Invasive Neurosurgery 53(3):147152, 2010 doi:10.1055/s-0030-1254145

10. Choi KY, Eun SS, Lee SH, Lee HY: Percutaneous endoscopic thoracic discectomy; transforaminal approach. Minimally Invasive Neurosurgery 53(1):25-28, 2010 doi:10.1055/s-0029-1246159

11. Choi KC, Park C-K: Percutaneous endoscopic lumbar discectomy for L5-S1 disc herniation: Consideration of the relation between the iliac crest and L5-S1 disc. Pain Physician 18:301-308, 2016

12. Fanous AA, Tumialán LM, Wang MY: Kambin's triangle: Definition and new classification schema. Journal of Neurosurgery Spine 32:390-398, 2020

13. Heo DH, Lee Dk, L. DC, Kim HS, Park CK: Fully endoscopic 
transforaminal lumbar discectomy for upward migration of upper lumbar disc herniation: Clinical and radiological outcomes and technical considerations. Brain Sciences 10:1-9, 2020

14. Hoogland T, Schubert M, Miklitz B, Ramirez A: Transforaminal posterolateral endoscopic discectomy with or without the combination of a low-dose chymopapain: A prospective randomized study in 280 consecutive cases. Spine (Phila Pa 1976), 31(24):E890E897, 2006 doi:10.1097/01.brs.0000245955.22358.3a

15. Kambin P, Sampson S: Posterolateral percutaneous suctionexcision of herniated lumbar intervertebral discs. Report of interim results. Clinical Orthopaedics and Related Research 207:37-43, 1986

16. Kim HS, Park JY: Comparative assessment of different percutaneous endoscopic interlaminar lumbar discectomy (PEID) techniques. Pain Physician 16(4):359-367, 2013

17. Kim HS, Adsul N, Kapoor A, Choi SH, Kim JH, Kim KJ, et al.: A Mobile Outside-in Technique of Transforaminal Lumbar Endoscopy for Lumbar Disc Herniations pp1-7:2018

18. Kim HS, Ju CI, Kim SW, Kim JG: Endoscopic transforaminal suprapedicular approach in high grade inferior migrated lumbar disc herniation. Journal of Korean Neurosurgical Society 45(2): 67-73, 2009 doi:10.3340/jkns.2009.45.2.67

19. Kim HS, Paudel B, Jang JS, Lee K, Oh SH, Jang IT: Percutaneous endoscopic lumbar discectomy for all types of lumbar disc herniations $(\mathrm{LDH})$ including severely difficult and extremely difficult LDH cases. Pain Physician 21(4):E401-E408, 2018

20. Kim HS, You JD, Ju CI: Predictive scoring and risk factors of early recurrence after percutaneous endoscopic lumbar discectomy. BioMed Research International pp9-12, 2019 doi:10.1155/2019/ 6492675

21. Lee S, Kim SK, Lee SH, Kim WJ, Choi WC, Choi G, Shin SW: Percutaneous endoscopic lumbar discectomy for migrated disc herniation: Classification of disc migration and surgical approaches. European Spine Journal 16(3):431-437, 2007 doi:10.1007/ s00586-006-0219-4

22. Liu C, Chu L, Yong HC, Chen L, Deng ZL: Percutaneous endoscopic lumbar discectomy for highly migrated lumbar disc herniation. Pain Physician 20(1):E75-E84, 2017

23. Ren Y, Yang J, Chen CM, Liu K, Wang XF,. . . Chu L: Outcomes of discectomy by using full-endoscopic visualization techni- que via the transcorporeal and transdiscal approaches in the treatment of cervical intervertebral disc herniation: A comparative study. BioMed Research International 2020, 5613459. doi:10.1155/ 2020/5613459

24. Ruetten S, Komp M, Godolias G: A New full-endoscopic technique for the interlaminar operation of lumbar disc herniations using 6-mm endoscopes: Prospective 2-year results of 331 patients. Minimally Invasive Neurosurgery 49(2):80-87, 2006 doi:10.1055/ s-2006-932172

25. Ruetten S, Komp M, Merk H, Godolias G: Full-endoscopic cervical posterior foraminotomy for the operation of lateral disc herniations using 5.9-mm endoscopes: A prospective, randomized, controlled study. Spine (Phila Pa 1976), 33(9):940-948, 2008 doi:10.1097/BRS.0b013e31816c8b67

26. Schubert M, Hoogland T: Endoscopic transforaminal nucleotomy with foraminoplasty for lumbar disk herniation. Operative Orthopädie und Traumatologie 17(6):641-661, 2005 doi:10.1007/ s00064-005-1156-9

27. Shin BJ: Risk factors for recurrent lumbar disc herniations. Asian Spine Journal 8(2):211-215, 2014 doi:10.4184/asj.2014.8.2.211

28. Wang H, Zhou Y, Li C, Liu J, Xiang L: Risk factors for failure of single-level percutaneous endoscopic lumbar discectomy. Journal of Neurosurgery: Spine 23(3):320-325, 2015 doi:10.3171/2014. 10.SPINE1442

29. Wang Y, Ma D, Tan W, Lai J: Combining YESS and TESSYS techniques during percutaneous transforaminal endoscopic discectomy for multilevel lumbar disc herniation. Med (United States), 97, 2018

30. Xin G, Shi-Sheng H, Hai-Long Z: Morphometric analysis of the YESS and TESSYS techniques of percutaneous transforaminal endoscopic lumbar discectomy. Clinical Anatomy 26(6):728734, 2013 doi:10.1002/ca.22286

31. Yeung AT, Tsou PM: Posterolateral endoscopic excision for lumbar disc herniation: Surgical technique, outcome, and complications in 307 consecutive cases. Spine (Phila Pa 1976), 27(7): 722-731, 2002 doi:10.1097/00007632-200204010-00009

32. Yin S, Du H, Yang W, Duan C, Feng C, Tao H: Prevalence of recurrent herniation following percutaneous endoscopic lumbar discectomy: A meta-analysis. Pain Physician 21(4):337-350, 2018 